\title{
Role of Abdominal Ultrasonography in the Early Diagnosis of Neonatal Necrotizing Enterocolitis
}

\author{
Shymaa F. Ibrahim*, Mohammed A. Mohammed*, Hesham A. Amen** and \\ Ashraf M. Radwan* \\ Departments of Pediatrics* and Radiology**, Faculty of Medicine, Sohag University
}

\section{Abstract}

Introduction: Necrotizing enterocolitis (NEC) can be defined as inflammatory intestinal disease characterized by variable degree of damage to the intestinal tract, ranging from mucosal injury to full-thickness necrosis and perforation (Obladen 2009).

Aim of the work: to evaluate patients with suspected or proven NEC by abdominal and Doppler ultrasound to examine the usefulness of abdominal ultrasound and color Doppler ultrasound in early diagnosis of Necrotizing Enterocolitis.

Patients and Methods: Prospective hospital based study that is conducted at Neonatal intensive care unit (N.I.C.U) at Sohag University Hospital. The study Period is from $31^{\text {th }}$ October 2015 to $30^{\text {th }}$ April 2016. All neonates with clinical, radiological findings suggesting necrotizing enterocolitis were included in the study.

Results: A total of 20 neonates had met the inclusion criteria. Patients had variable clinical presentation, most common presentation was abdominal distension and brownish vomiting $35 \%$ of patients; $80 \%$ of patients had tense abdomen; $80 \%$ of patients had dullness of the abdomen; $80 \%$ had inaudible intestinal sounds and 20\% had sluggish intestinal sounds. By Abdominal US Pneumatosis intestinalis was detected in 50\% of cases in which $30 \%$ were diffuse and $20 \%$ were segmental in contrast to X ray that detected only $35 \%$ of cases ( $\mathrm{p}$ value 0.003 ). Portal venous gas was detected in $25 \%$ of cases by US in comparison to only $10 \%$ by $\mathrm{X}$ ray with $\mathrm{p}$ value of 0.001 .

Conclusion: Abdominal ultrasound and color Doppler ultrasound were helpful in detecting cases with early stage of NEC. Abdominal ultrasound was helpful in early detection of complication as intestinal perforation and so early surgical management; thus may decrease morbidity and mortality rates.

Keywords: Necrotizing Enterocolitis, neonates, ultrasound, color Doppler.

\section{Introduction}

Necrotizing enterocolitis (NEC) is as inflammatory intestinal disease characterized by variable degree of damage to the intestinal tract, ranging from mucosal injury to full-thickness necrosis and perforation (Obladen 2009). It is the most common lifethreatening gastrointestinal emergency in the Neonatal Intensive Care Unit (NICU) especially in premature infant (Henry et al. 2009).

The incidence of NEC is correlated to gestational age and birth weight. Low birth weight, premature neonates are affected as high as $15 \%$ of all neonates cared for in the NICU (Henry et al. 2009).

The etiology of necrotizing enterocolitis is multifactorial. Prematurity is the most predisposing factor for necrotizing enterocolitis and it rarely occurs in full-term infants, where it is usually secondary to congenital diseases, such as cardiac anomalies (Bolisetty et al. 2000). Hypoxia can lead to necrotizing enterocolitis: recurrent episodes of apnea, respiratory distress, assisted ventilation, and umbilical vessel catheterization (Palmer et al. 1987). 
Disturbance of commensal bacteria, leading to microbial colonization of the gut, has been implicated in the pathogenesis of necrotizing enterocolitis. The most commonly identified organisms are: Escherichia Coli, Klebsiella pneumonia; Proteus, Staphylococcus aureus, S. Epidermis, Enterococcus spp (Patel et al. 2015).

The most common presenting sign is abdominal distension, which is frequently accompanied by bilious vomiting and feeding intolerance with gastric aspirates. A decrease in urinary output and poor perfusion may also be noted. Late findings of peritonitis include abdominal wall edema, erythema.

\section{Aim of the work:}

In this study we aimed to evaluate patients with suspected or proven NEC by abdominal and Doppler ultrasound to examine the usefulness of abdominal ultrasound and color Doppler ultrasound in early diagnosis of Necrotizing Enterocolitis.

\section{Patients and Methods:}

\section{Study:}

Prospective hospital based study that was conducted at Neonatal intensive care unit (N.I.C.U) at Sohag University Hospital in the period from $31^{\text {th }}$ October 2015 to $30^{\text {th }}$ April 2016.

\section{Patients:}

All neonates with clinical, radiological findings suggesting necrotizing enterocolitis were included in the study.

\section{Methods:}

All neonates included in the study will be subjected to a detailed clinical history of the patients, detailed examination focusing on gastrointestinal system. Abdominal Ultrasonography, simple plain erect X-ray abdomen and Doppler ultrasonography were done on the day of their diagnosis and on the third day after diagnosis. Complete blood count (CBC), electrolytes (Na, K), CRP were done.

\section{Ethical Considerations}

Approval of Sohag Faculty of Medicine Research Ethics Committee, Verbal consent was taken from guardians.

\section{Statistical analysis:}

- Statistical package for social sciences (IBM-SPSS), version 24 IBM- Chicago, USA (May 2016) was used for statistical data analysis.

- Data expressed as mean, standard deviation (SD), number and percentage. Mean and standard deviation were used as descriptive value for quantitative data, while number and percentage were used to describe qualitative data.

- Student $t$ test was used to compare the means between two groups, and Pearson Chi square was used to compare percentages of qualitative data.

- The level of significance (P-value) can be explained as:
$\circ$ No significance $\mathrm{P}>0.05$
$\circ$ Significance $\mathrm{P}<0.05$
○ High significance $\mathrm{P}<0.001$

\section{Results}

A total of 20 neonates ( $80 \%$ were preterm and $20 \%$ were full term) had met the inclusion criteria in which $45 \%$ of cases were male and $55 \%$ were female. The mean age at presentation of study group was $16.9 \pm 8.7$ days (range 2-36 days). The mean gestational age of study group was 33.2 \pm 2.5 week (range $30-38$ week). The mean 
weight of study group was $1.4 \pm 0.75 \mathrm{~kg}$ (range $0.6-2.3 \mathrm{~kg}$ ) and the risk factors for NEC in full term were congenital heart disease, hypoxic ischemic disease, neonatal sepsis, exchange transfusion and patients with small weight for gestational age.

Patients had variable clinical presentation, most common presentation was abdominal distension and brownish vomiting $35 \%$ of patients; $80 \%$ of patients had tense abdomen; $80 \%$ of patients had dullness of the abdomen; $80 \%$ had inaudible intestinal sounds and $20 \%$ had sluggish intestinal sounds.

There was an association between NEC and neonatal sepsis as all cases had elevated marker of sepsis as CRP (mean 53.1 \pm 48.3 ), total leucocyte count (mean $11.9 \pm 10.1$ ). Most cases had thrombocytopenia as mean platelet count of study group was 84.6 \pm 71.9 . Mean Serum sodium of study group was $134.2 \pm 4.8$ and the mean serum potassium was $4.09 \pm 0.68$.

By abdominal radiograph, all cases show intestinal dilatation, $90 \%$ of cases had diffuse dilatation and $10 \%$ had segmental dilatation. Free air was found in $25 \%$ of cases. Mural air detected in 35\% of cases in which 30\% of cases were diffuse and 5\% were segmental. Portal venous gas was found in $15 \%$ of cases. Air fluid level was found in $5 \%$ of cases.

By Abdominal US, 95\% of cases had diffuse dilatation and 5\% had segmental dilatation; $60 \%$ of cases had sluggish intestinal motility and $40 \%$ of cases had immotile loops. Free air was found in $20 \%$ of cases. Mural air detected in $55 \%$ of cases in which $30 \%$ were diffuse and $25 \%$ were segmental. Ascites was detected in all cases in which $20 \%$ were turbid. Portal venous gas was found in $30 \%$ of cases.

Doppler finding, as regard intestinal wall vascularity $55 \%$ of cases had hypo vascular intestinal wall $35 \%$ of cases had hyper vascular intestinal wall and only $10 \%$ were normal. Mean resistance index of SMA of study group was $0.77 \pm 0.12$

By Abdominal US Pneumatosis intestinalis was detected in $50 \%$ of cases in which $30 \%$ were diffuse and $20 \%$ were segmental in contrast to $\mathrm{X}$ ray that detected only $35 \%$ of cases ( $p$ value 0.003 ). Portal venous gas was detected in $25 \%$ of cases by US in comparison to only $10 \%$ by $\mathrm{X}$ ray with p value of 0.001 .

\section{Discussion}

In the current study patients had variable clinical presentation, abdominal distension and brownish vomiting was the most common presentation. These results were in agreement with the results of study done by (Kim et al. 2005) who reported that the most common signs among NEC patients were gastric residua in all patients $(100 \%)$, abdominal distention (60\%), vomiting (15\%), and bloody stool (10\%).
On examination $80 \%$ of patients had tense tender abdomen, $80 \%$ of patients had dullness abdomen, $80 \%$ of patients had inaudible intestinal sounds, and $20 \%$ had sluggish intestinal sounds, these results were near to the results of the study done by (Deeg et al. 1987) who reported in his study that protruding tender abdominal wall was found in 14 neonates $(100 \%)$, bloody stool in 11neonates (79\%), erythema of the abdominal wall in 9 neonates $(64 \%)$. 
There was an association between NEC and neonatal sepsis as all cases had elevated marker of sepsis as CRP and Total leucocyte count. These results were close to the results of study done by (Muchantef et al. 2013) who reported in his study that patients with NEC were associated with sepsis as CRP mean was 14.9 and total leucocyte count mean was 15.4.

In the current study most cases had thrombocytopenia, this was in agreement with the result of study done by (Hashem et al. 2017) who reported that NEC cases had thrombocytopenia in which mean platelet count was $80.16 \pm 31.89$.

By abdominal ultrasound Intestinal dilatation was the most frequently detected abnormality in neonates with suspected or proven $\mathrm{NEC}$, in the present study intestinal dilatation detected in all cases, that was similar to (Monica Epelman et al. 2007) study who revealed that intestinal dilatation was present in over $90 \%$ of patients, with the remaining $10 \%$ showing only minor or nonspecific disturbances of bowel gas pattern.

Portal venous gas (PVG) was detected in $25 \%$ of cases by AUS compared to $10 \%$ of cases with portal venous gas detected by abdominal radiograph so AUS more sensitive than abdominal radiograph in detecting PVG, these results were near to the results of study done by (Monica Epelman et al. 2007) who revealed in his study that $26 \%$ of cases were detected by AUS.

\section{Conclusion:}

Abdominal ultrasound and color Doppler ultrasound were helpful in detecting cases with early stage of NEC. Abdominal ultrasound was helpful in early detection of complication as intestinal perforation and so early surgical management; thus may decrease morbidity and mortality rates.

\section{References:}

1. Bolisetty, S., K. Lui, J. Oei and J. Wojtulewicz (2000). "A regional study of underlying congenital diseases in term neonates with necrotizing enterocolitis." Paediatrica 89(10): 1226-1230.

2. Deeg, K., S. Wehner, T. Rupprecht and H. Singer (1987). "pulsed doppler sonography in the anterior cerebral-arteries and the truncus coeliacus in infants with coarctation of the aorta in comparison to a healthy control-group." klinische padiatrie 199(6): 411-423.

3. Hashem, R. H., Y. A. Mansi, N. S. Almasah and S. Abdelghaffar (2017). "Doppler ultrasound assessment of the splanchnic circulation in preterms with neonatal sepsis at risk for necrotizing enterocolitis." $\quad \underline{J}$ Ultrasound 20(1): 59-67.

4. Henry, M. C. and R. L. Moss (2009). "Necrotizing enterocolitis." Annu Rev Med 60: 111-124.

5. Kim, W. Y., W. S. Kim, I. O. Kim, T. H. Kwon, W. Chang and E. K. Lee (2005). "Sonographic evaluation of neonates with early-stage necrotizing enterocolitis." Pediatr Radiol 35(11): 1056-1061.

6. Monica Epelman, M., A. Daneman, O. M. Navarro, M. Faingold, G. Taylor and J. T. Gerstle (2007). "Necrotizing Enterocolitis: Review of State-of-the-Art Imaging Findings with Pathologic Correlation1."

7. Muchantef, K., M. Epelman, K. Darge, H. Kirpalani, P. Laje and S. A. Anupindi (2013). "Sonographic and radiographic imaging features of the neonate with necrotizing enterocolitis: correlating findings 
with outcomes." Pediatr Radiol 43(11): 1444-1452.

8. Obladen, M. (2009). "Necrotizing enterocolitis-150 years of fruitless search for the cause." Neonatology 96(4): 203-210.

9. Palmer, S., S. Thomas, R. Cooke, D. Low, W. Fysh, J. Murphy, G. Gandy and H. Gamsu (1987).
"Birthweight-specific risk factors for necrotising enterocolitis." Journal of Epidemiology \& Community Health 41(3): 210-214.

10. Patel, R. M. and P. W. Denning (2015). "Intestinal microbiota and its relationship with necrotizing enterocolitis." Pediatric research 78(3): 232. 